\title{
A FUNCTIONAL-ANALYTIC MODEL OF ANALOGY USING THE RELATIONAL EVALUATION PROCEDURE
}

\author{
IAN STEWART \\ National University of Ireland, Galway \\ DERMOT BARNES-HOLMES and BRYAN ROCHE \\ National University of Ireland, Maynooth
}

\begin{abstract}
Analogical reasoning is conceptualized by Relational Frame Theory as responding in accordance with an equivalence relation between equivalence or other types of derived stimulus relations. The purpose of this study was to provide an empirical demonstration of analogy using the Relational Evaluation Procedure (REP), a recently developed technique for the rapid training and testing of derived stimulus relations. The experiment involved 9 stages in which 5 adult male subjects were exposed to a complex series of REP training and testing protocols, by the end of which they each readily demonstrated 24 completely novel instances of responding in accordance with analogical relations as conceptualized by RFT. The implications of these results for future functional analytic investigations of analogical reasoning are discussed.
\end{abstract}

Analogy is undoubtedly an important feature of human language and cognition. Apart from its use in the elucidation of novel concepts in such fields as science, technology, and education, for example, it is also a ubiquitous aspect of everyday human communication. Unsurprisingly, therefore, there has been extensive psychological research devoted to understanding and modeling this phenomenon.

Theoretical and empirical analysis of analogical language has traditionally been seen as the preserve of the cognitivist approach (e.g., Vosniadou \& Ortony, 1989). From this perspective, analogy has most often been understood in terms of the mapping of relational information from one domain (the base domain) to another (the target domain). Eysenck and Keane (2000) list several symbolic representational models that incorporate these characteristics, including the Structure Mapping Engine (e.g., Forbus, Ferguson, \& Gentner, 1994), the Incremental Analogy Machine (e.g., Keane, Ledgeway, \& Duff, 1994), and the Analogical Constraint Mapping Engine (Holyoak \& Thagard, 1989). More recent cognitivist research has begun to produce connectionist models of analogy (e.g., LISA [Learning and Inference based on Schemas and

Correspondence may be sent to Ian Stewart, National University of Ireland, Galway, Ireland. (E-mail: ian.stewart@nuigalway.ie). 
Analogies; Hummel \& Holyoak, 1997]; DRAMA [Distributed Representation Analogy MApper; Eliasmith \& Thagard, 2001]), which are seen as more flexible with regard to semantic content as well as more neurologically plausible. From a behavior analytic perspective, however, a key weakness shared by both the newer connectionist as well as the earlier representational models is that they define analogical reasoning using information processing concepts such as 'mapping' and 'knowledge transfer' that remain ill-defined within the behavioral tradition. Furthermore, though the new generation of connectionist models appear to have certain important advantages over their predecessors, it is arguable that they are more interesting as models of neurological rather than of psychological functioning.

Until relatively recently, behavior analysis provided little in the way of an alternative to this cognitivist approach. The main contribution was the relatively early, and largely interpretive work of B. F. Skinner (1957). For Skinner, analogy was a form of 'metaphorical extension,' which was itself classified as a subtype of the 'extended tact.' The tact is a behavioral relation that is defined as "a verbal operant in which a response of a given form is evoked (or at least strengthened) by a particular object or event or property of an object or event" (pp. 81-82). The extended tact is a feature of more complex verbal behavior that occurs when a response is evoked by a novel stimulus that resembles a stimulus previously present when a response was reinforced. Metaphorical verbal behavior is a subtype of this latter class of behavior that takes place "because of the control exercised by properties of the stimulus which, though present at reinforcement, do not enter into the contingency respected by the verbal community" (p. 92). The following is an example of the Skinnerian interpretation of metaphorical extension that appears in 'Verbal Behavior' (1957):

When for the first time a speaker calls someone a mouse, we account for the response by noting certain properties-smallness, timidity, silent movement and so on-which are common to the kind of situation in which the response is characteristically reinforced and to the particular situation in which the response is now emitted. Since these are not the properties used by zoologists or by the lay community as the usual basis for reinforcing a response we call the extension metaphorical. (p.93)

A similar description of the phenomenon of metaphorical extension appears in 'Recent Issues in the Analysis of Behavior' (1989):

When we speak of weighing evidence [for example] we are using a metaphor. But a metaphor is a word that is "carried over" from one referent to another on the basis of a common property. The common property in weighing is the conversion of one kind of thing (potatoes or evidence) into another (a number on a scale or a verdict). Once we have seen this kind of thing done with potatoes it is easier to see it done with evidence ... We could also say that 


\begin{abstract}
weight becomes abstract when we move from potatoes to evidence. The word is indeed abstracted in the sense of its being drawn away from its original referent, but it continues to refer to a common property, and, as in the case of metaphor, in a possibly more decisive way. The testimony in a trial is much more complex than a sack of potatoes, and "guilty" probably implies more than "ten pounds." But abstraction is not a matter of complexity. Quite the contrary. Weight is only one aspect of a potato, and guilt is only one aspect of a person. Weight is as abstract as guilt. It is only under verbal contingencies of reinforcement that we respond to single properties of things or persons. In doing so we abstract the property from the thing or person. (p. 7)
\end{abstract}

In these quotations, Skinner essentially conceptualizes analogy as the abstraction, via one particular subtype of verbal behavior (i.e., the extended tact), of a common physical property from two different types of environmental event. This conceptualization undoubtedly provides a useful starting point for the analysis of analogical language. However, supplementary accounts of the behavioral processes underlying this phenomenon are required. For example, how does a repertoire of analogical verbal behavior, such as "A is to B as C is to D," develop from a (presumably) simpler repertoire of formal property abstraction? This is one important question left unanswered by the Skinnerian analysis. Furthermore, this analysis is interpretational only. Empirical analyses of analogy are required in order to provide a more complete behavior analytic treatment of this phenomenon.

More recent behavior analytic work has attempted to provide such analyses. The theoretical background to this work is Relational Frame Theory (RFT; Hayes, Barnes-Holmes, \& Roche, 2001), a modern, explicitly functional analytic approach to language and cognition that investigates these phenomena as examples of derived relational responding (DRR). The most well researched example of DRR is equivalence responding. For example, a person might be trained to choose $A$ in the presence of $B$ and $B$ in the presence of $C$ and might subsequently demonstrate the untrained responses of choosing $B$ in the presence of $A, C$ in the presence of $B, A$ in the presence of $C$, and $C$ in the presence of $A$. These responses are evidence that the person is now relating the training stimuli as equivalent to each other. In other words, based on the training, a subject derives a relation of equivalence between the stimuli involved. Relational Frame Theory sees equivalence as a basic and prototypical form of DRR, claiming that it is particularly important in the analysis of language in that it appears to provide a laboratory analog of linguistic reference. At the same time, equivalence is only one example of the phenomenon of DRR. Other examples that have been demonstrated in the behavioral laboratory include responding in accordance with relations of opposition, distinction, and comparison. Steele and Hayes (1991), for example, provided an empirical demonstration of responding in accordance with relations of equivalence 
and difference. In one experiment, subjects were pretrained to relate physically identical stimuli in the presence of an arbitrary stimulus, referred to as the contextual cue of SAME and to relate physically different stimuli in the presence of a second contextual cue (DIFFERENT). The researchers then trained and tested subjects in a number of related conditional discriminations, with each discrimination taking place in the presence of one of the two contextual cues. For example, having been trained to relate $\mathrm{A} 1$ to $\mathrm{C} 1$ in the presence of SAME, and $A 1$ to $B 2$ in the presence of DIFFERENT, subjects subsequently related $\mathrm{B} 2$ to $\mathrm{C} 1$ when presented with the DIFFERENT cue. More recent research has replicated and extended this early work (e.g., Dymond \& Barnes, 1994, 1995, 1996; Roche \& Barnes, 1996).

Recently, RFT researchers have begun to provide derived relational responding based models of analogy. Barnes, Hegarty, and Smeets (1997) provided the first functional-analytic RFT-based model of analogical reasoning as responding in accordance with equivalence relations between equivalence relations. In the authors' own words:

Consider ... the following question based on the classic proportion scheme (A : B :: C : ?); "apple is to orange as dog is to: (i) sheep, or (ii) book?". If "apple" and "orange" participate in an equivalence relation in the context "fruit," and "dog" and "sheep" participate in an equivalence relation in the context "animals" then we would expect a person to pick "sheep" as the correct answer. In effect, the response would be in accordance with the derived equivalence relation between two already established separate equivalence relations. . . We take the view that equivalence-equivalence responding is an example of a relational network as defined by relational frame theory (e.g., Barnes, 1994; Barnes \& Holmes, 1991; Hayes, 1991, 1994) (p. 3).

In the study reported by Barnes et al. (1997), subjects were trained, using a standard matching-to-sample format, to make the following conditional discriminations: $A 1 \rightarrow B 1, A 2 \rightarrow B 2, A 1 \rightarrow C 1, A 2 \rightarrow C 2, A 3 \rightarrow B 3, A 3 \rightarrow C 3$, $A 4 \rightarrow B 4, A 4 \rightarrow C 4$. Four equivalence relations then emerged: $B 1 \leftrightarrow C 1$, $\mathrm{B} 2 \leftrightarrow \mathrm{C} 2, \mathrm{~B} 3 \leftrightarrow \mathrm{C} 3, \mathrm{~B} 4 \leftrightarrow \mathrm{C} 4$. Tests were then conducted to determine whether equivalence relations between equivalence relations (e.g., B1C1↔B3C3) and equivalence relations between nonequivalence relations (e.g., $\mathrm{B} 1 \mathrm{~B} 2 \leftrightarrow \mathrm{C} 3 \mathrm{C} 4)$ would emerge. Results showed that a range of subjects did indeed demonstrate this 'equivalence-equivalence' responding.

Stewart, Barnes-Holmes, Roche, \& Smeets (2001) recently extended this initial model in accordance with Skinner's (1957) interpretation of analogy. Specifically, these authors argued that in addition to the arbitrary relations established by Barnes et al. (1997), analogy often involves the abstraction of common formal properties (see Skinner's discussion of 'metaphorical extension,' 1957, p. 92). In the example given above, for instance, the arbitrary equivalence relation between the words "apple" and "orange" is based, to some degree, on the nonarbitrary or physical 
relation of similarity between actual apples and actual oranges (i.e., both are small, spherical, edible, sweet, etc.). Similarly, the arbitrary equivalence relation between the words "dog" and "sheep" is based on the nonarbitrary relation of similarity between actual dogs and actual sheep (i.e., in general, they are four legged, mobile, hairy, etc). Thus, the equivalence-equivalence or analogical relation between the equivalence relations 'apple-orange' and 'dog-sheep' may be traced back to the formal relations that obtain between particular objects in the environment. Stewart, Barnes-Holmes, Roche, et al. (2001), therefore, attempted to include the role of formal properties in the Barnes et al. (1997) model.

Subjects were taught, using a delayed matching-to-sample procedure, to choose a particular nonsense syllable in the presence of each of four blue and four red geometric shapes. In a subsequent test, subjects demonstrated equivalence responding based on the abstraction of color by consistently matching nonsense syllables related to same-colored shapes to each other. Subjects then showed equivalence-equivalence responding in which equivalence relations from the previous part of the experiment were related to other equivalence relations, and nonequivalence relations were related to other nonequivalence relations. Thus, these researchers provided a demonstration of equivalence-equivalence responding based on the abstraction of common formal properties, thereby extending the functionalanalytic model of Barnes et al. (1997) to incorporate what appears to be an important feature of analogy.

Both the Stewart et al. (2001) and Barnes et al. (1997) studies trained and tested subjects using the matching-to-sample procedure (see also Stewart, Barnes-Holmes, Roche, \& Smeets, 2002). One possible criticism of this procedure, however, is that the extensive training and testing involved in this procedure results in the demonstration of a limited number of analogies (i.e., only those that are possible based on the trained and tested equivalence relations). A relatively new methodology might solve this problem, however. Following the empirical demonstration of multiple stimulus relations in a number of studies, Hayes and Barnes (1997) argued for the need to develop new methodologies so that multiple stimulus relations might be analyzed and experimentally manipulated more readily than is possible with the traditional MTS procedure. One methodology that they suggested as suitable for this work was the Relational Evaluation Procedure (REP; see also Hayes et al., 2001; Cullinan, Barnes, \& Smeets, 1998; Cullinan, Barnes-Holmes, \& Smeets, 2000). The core feature of the REP is that it allows subjects to evaluate, or report on, the stimulus relation or relations that are presented on a given trial. In the typical approach, subjects may confirm or deny the applicability of particular stimulus relations to other sets of stimulus relations. For example, on a typical trial, a subject might be presented with a contextual cue for DIFFERENT and two arbitrary stimuli that are specified within that trial as participating in a difference relation. The subject is then required to choose between two arbitrary shapes for which the response functions of TRUE and FALSE have previously been 
established. In this case, the subject should choose TRUE, because the DIFFERENT cue coordinates with the arbitrary stimulus relation. If the cue had been SAME or the stimulus relation had been one of sameness, the appropriate choice would have been FALSE. In pilot studies using this methodology, it has been found that once a number of appropriate contextual cues have been established, a potentially infinite number of relational responses may be observed. The critical point is that the number of relational responses that may be observed is not constrained by the prior training and testing of a specific set of derived relations. Given the apparent power of this methodology to generate unconstrained numbers of derived stimulus relations, it might now be adopted by researchers exploring a number of disparate areas. In the context of the current study, the REP may be useful in the analysis of relating relations, which RFT views as the key characteristic of analogical and metaphorical language (Stewart et al., 2002). In the current article, we describe how we have used the REP to analyze the relating of relations. As in the example provided above, contextual cues are presented with stimulus relations, and TRUE and FALSE response options. However, in the present study, the critical arbitrary stimulus relations are relations between stimulus relations rather than between individual stimuli. For example, if a SAME cue is presented with two stimulus pairs, each of which participates in a difference relation, the appropriate choice in this case is TRUE, because the relations between the two pairs are the same.

\section{Method}

\section{Subjects}

Five subjects, all Caucasian males, participated in this experiment. The ages of the subjects ranged from 19 to 31 years (mean $=25$ ). One of the subjects was a non-psychology undergraduate student, two were non-psychology graduate students, and the remaining two were nonacademic professionals. All subjects were recruited through personal contacts and none of them had any prior experience of research in the areas of derived stimulus relations or RFT.

Subjects were paid at the rate of 3 Irish pounds (4 U.S. dollars) an hour. They were given 1 Irish penny (1.3 U.S. cents) for every correct response, and lost 1 penny for every incorrect response. They were also paid 20 Irish pounds (27 U.S. dollars) for completing the entire experiment. Subjects were informed of these conditions by the experimenter before they agreed to participate in the study.

All subjects were exposed to the experimental procedures individually. If a subject did not complete the experiment in one session, then he or she was asked to return on a subsequent day (usually the following day). To ensure that the previously established performances were still intact, at the beginning of the next session the subject was reexposed to those stages of the experiment that he had previously completed. On some occasions, therefore, a subject could successfully 
complete a particular stage in the experiment, but would be reexposed to that stage for a second time. All subjects were asked not to discuss their participation in the experiment with anyone, and sessions were arranged so that subjects did not meet each other in the vicinity of the laboratory. When the experiment was finished subjects were thanked, debriefed, and paid the appropriate amount depending on their performance.

\section{Apparatus and Materials}

Each subject was seated in a small experimental room at a table on which was placed an Apple Macintosh ${ }^{\mathrm{TM}}$ Microcomputer (iMac) that displayed both colored stimuli and black characters on a white background. Stimulus presentation and the recording of responses were controlled by the computer, which was programmed using PsyScope TM, a graphical system for the design of psychology experiments (see Roche, Stewart, \& Barnes-Holmes, 1999). A response involved using the mouse to 'click' on a chosen stimulus.

Computer-generated stimuli. The experimental stimuli included 30 black and white pictures of various objects, 300 colored shapes, 200 nonsense syllables, 4 arbitrary shapes as contextual cues, a small box in the middle of the screen in which stimuli appeared, and an eight-chambered box (see subsequent figures) at the bottom of the screen in which stimuli appeared. Subjects never saw the same configuration of stimuli twice across stages or within a particular stage of training and testing.

\section{Procedure}

All 5 subjects were trained and tested individually during sessions that lasted between 40 and 145 min each. The maximum number of sessions required to complete the experiment was three. The experiment involved nine stages. If a subject failed any test stage during the study, he was reexposed to all previous experimental stages. It was decided prior to the study that if a subject failed any test five times across the nine stages, then his participation in the experiment would be terminated (this never occurred, however).

Stage 1(a): Same/different training with pictures. In this initial stage, subjects were first presented with the following instructions (to see instructions for any subsequent stages, the reader should consult Appendix 1):

In a moment some images will appear on this screen. First, a box will appear at the bottom of the screen. Next, another image will appear above that. Then, another image will appear above that, and finally, two images will appear in the corners at the top of the screen. You should look first at the image that appears above the box. Next, look at the image that appears above that. Then, having looked at these two images, choose one of the two images in the top corners of the screen. Click the mouse when you are ready to begin.

After subjects clicked on the mouse, the screen cleared for $1.5 \mathrm{~s}$. The first stimulus then appeared $2 \mathrm{~cm}$ above the bottom of the screen. This 
stimulus may be described as follows: An eight-chambered box, approximately $5 \mathrm{~cm}$ by $20 \mathrm{~cm}$. Each chamber measured approximately $5 \mathrm{~cm}$ in height and $2.5 \mathrm{~cm}$ across. The eight-chambered box was outlined by another box (see Figure 1, upper panel). One second later, the contextual cue, an arbitrary black shape measuring approximately $1.5 \mathrm{~cm}$ by $3-4 \mathrm{~cm}$,
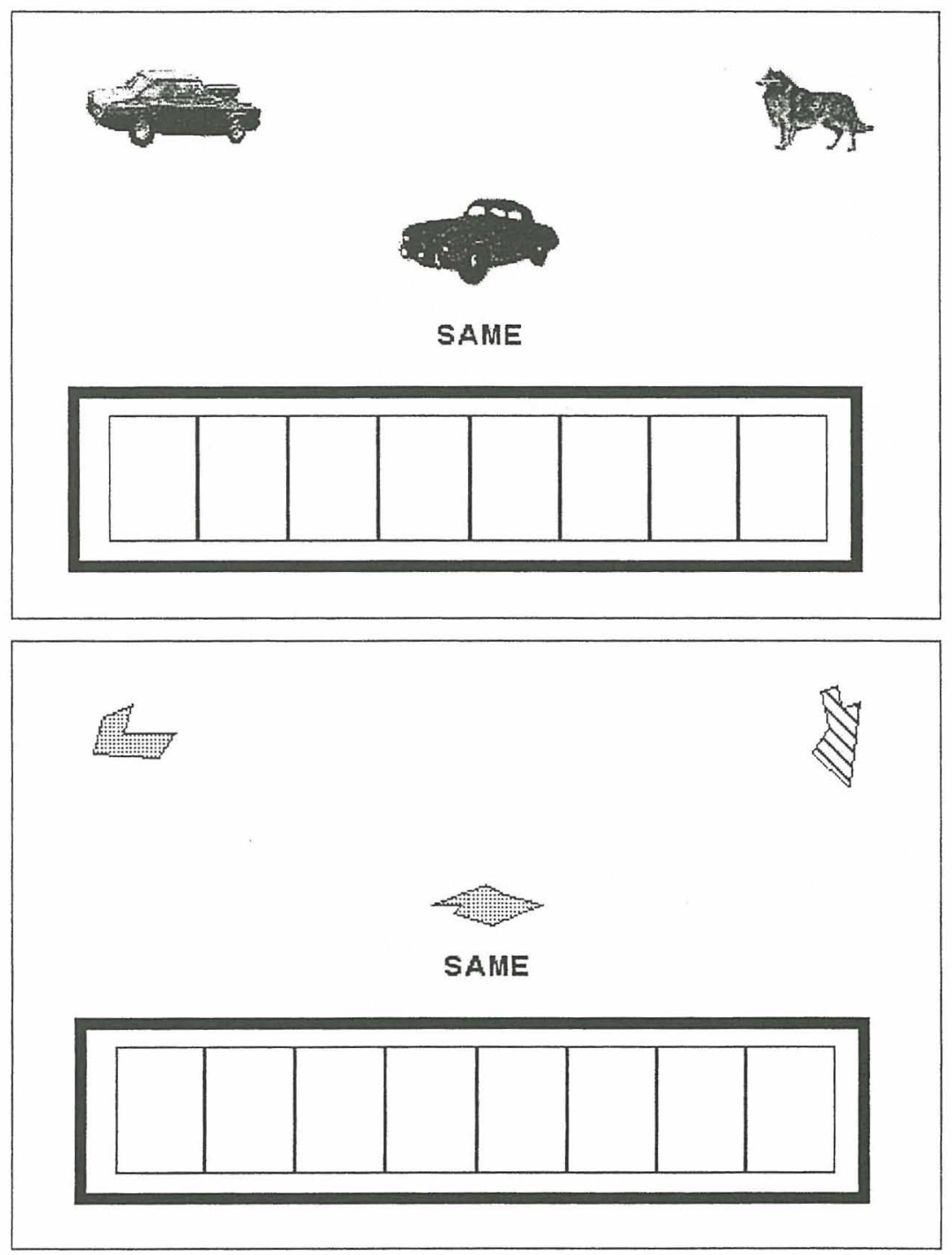

Figure 1. Upper panel: A trial-type representative of Stage 1(a \& b) SAME/DIFFERENT training and testing, which employed picture stimuli. The SAME and DIFFERENT contextual cues were arbitrary shapes, but are shown here as English words for ease of communication.

Lower panel: A trial-type representative of Stage 2(a \& b) training and testing, which employed colored shape stimuli. 
appeared approximately $2 \mathrm{~cm}$ above the center of the first stimulus. One second after that, the sample, a black and white picture measuring approximately $6 \mathrm{~cm}$ by $6 \mathrm{~cm}$, appeared approximately $3 \mathrm{~cm}$ above the contextual cue, and then, 1 further second after that, the comparisons, two black and white pictures approximately the same size as the sample picture, appeared in synchrony in the top corners of the screen.

On each trial, reinforcement was contingent upon choosing the comparison that was similar to the sample in the presence of one particular arbitrary shape (\%\%\%), and choosing the comparison that was different from the sample in the presence of the other arbitrary shape (!!!). This training was designed to establish the functions of SAME and DIFFERENT, respectively, in these arbitrary shapes, so that from this stage on, these shapes might be expected to serve as contextual cues for SAME and DIFFERENT responding, respectively. Subsequently, these shapes/contextual cues will be referred to as SAME and DIFFERENT, respectively.

Training was conducted in blocks of 12 trials. On each trial, the position of the comparison stimuli was varied randomly (i.e., the reinforced comparison could appear in either of the top corners of the screen with equal probability). Subjects chose a comparison by moving the cursor over the to-be-chosen comparison and then 'clicking' the mouse button. The correct completion of a training trial removed the stimulus display and produced the word "Correct $(+1 p)$ " in the center of the screen, accompanied simultaneously by a high pitched beep lasting approximately $1.5 \mathrm{~s}$. The incorrect completion of a training trial removed the stimulus display and produced the word "Wrong (-1p)" in the center of the screen accompanied simultaneously by a low, buzzing sound lasting approximately $1.5 \mathrm{~s}$. A 1-s intertrial interval (i.e., the screen cleared and remained blank) followed all programmed consequences. Only when the subject demonstrated 12 out of 12 correct responses within a block was he allowed to proceed to testing. Conditions of training for this stage, including numbers of trials per block, nature of feedback, length of intertrial interval and criterion for advancing to testing, applied also to all subsequent training stages.

Stage 1(b): Same/different testing with pictures. The same format was used in testing as in training. However, during the test the computer omitted all feedback messages and proceeded directly to the intertrial interval after the subject responded on each individual trial. Testing was conducted in blocks of 12 trials. If the subject failed to show 12 out of 12 correct responses, then he was retrained and retested. Only when the subject demonstrated 12 out of 12 correct responses in a single block of testing was he allowed to proceed to Stage 2(a). As stated previously, if the subject failed Stage 1(b), or any subsequent test stage, a total of five times then the subject's participation in the experiment was terminated (however, all subjects passed all stages within this five-exposure limit). Conditions of testing for this stage, including numbers of trials per block and criterion for passing, were the same for all subsequent testing phases, with the exception of Stage 9, which involved 24 test trials. 
Stage 2(a \& b): Same/different training and testing with colored shapes. Training and testing for Stage 2(a \& b) employed the same basic format and procedure as were used in Stage 1. In Stage 2, however, the sample and comparisons were colored shapes (rather than black and white pictures) and subjects had to choose the comparison that was the same color as the sample, irrespective of shape (see Figure 1, lower panel).

Stage 3(a \& b): True/false training. At the start of each trial, the first
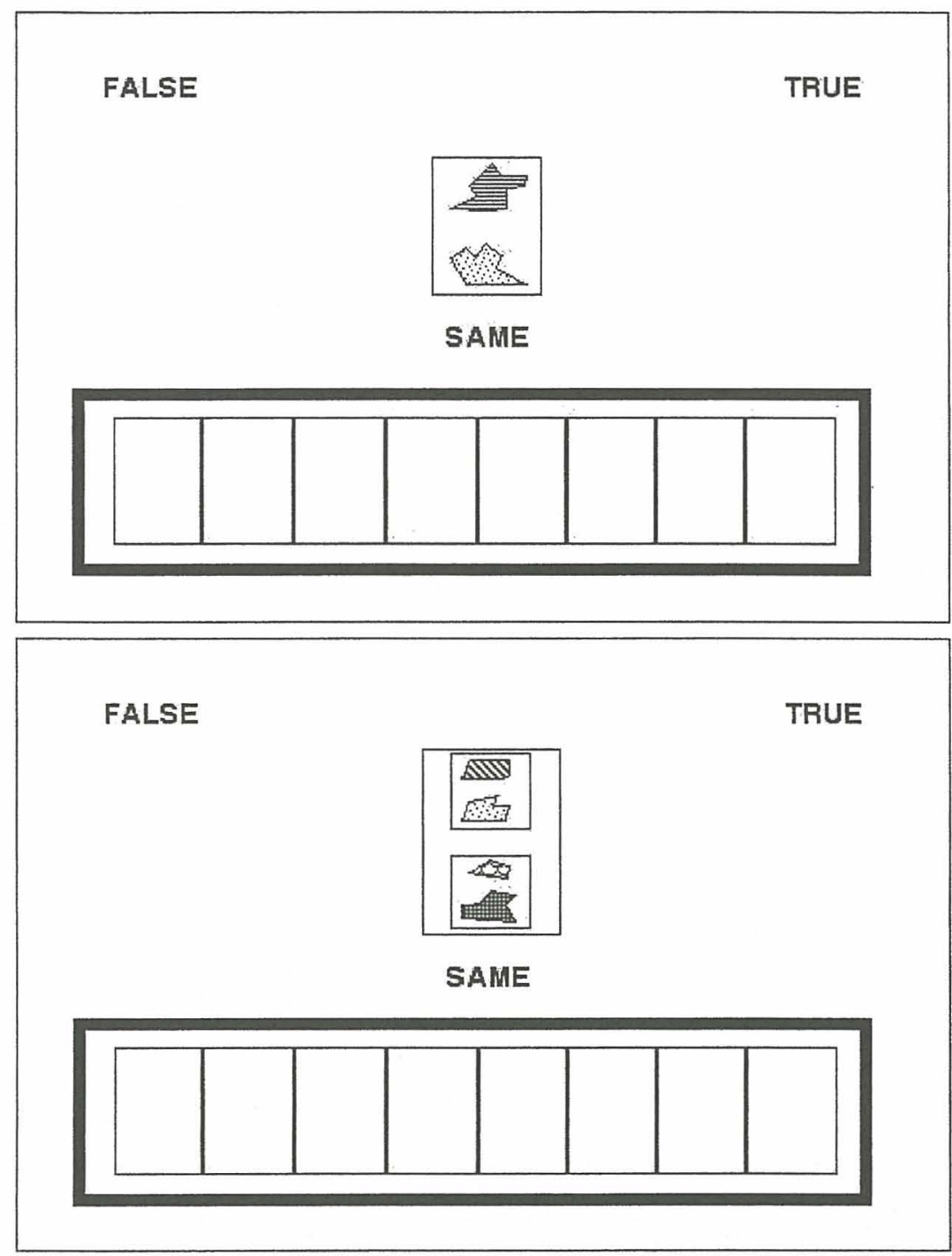

Figure 2. Upper panel: A trial-type representative of Stage 3(a \& b) YES / NO training and testing. The SAME, DIFFERENT, YES, and NO contextual cues were arbitrary shapes, but are shown here as English words for ease of communication.

Lower panel: A trial-type representative of Stage $4(\mathrm{a} \& \mathrm{~b})$ training and testing, the purpose of which was to establish the relating of (nonarbitrary color) relations. 
stimulus, the eight-chambered box, appeared $2 \mathrm{~cm}$ above the bottom of the screen (see Figure 2, upper panel). One second later, either SAME or DIFFERENT appeared approximately $2 \mathrm{~cm}$ above the center of the chambered box. Following another 1-s delay, the sample, a box measuring approximately $3 \mathrm{~cm}$ by $5 \mathrm{~cm}$ appeared approximately $3 \mathrm{~cm}$ above the contextual cue. Two colored shapes were located inside this box (see Figure 2, upper panel). Finally, 1s later, two arbitrary shapes, @ @ @ and ***, appeared in synchrony in the top corners of the screen. The respective position (i.e., left or right) of these stimuli was counterbalanced across trials, and this was the case for all subsequent stages.

On each trial, the sample was a box containing either two samecolored or two differently colored shapes. If the box contained two samecolored shapes, then in the presence of SAME, choosing@@@ was reinforced, whereas in the presence of DIFFERENT, choosing *** was reinforced. If the box contained two differently colored shapes, then in the presence of SAME, choosing *** was reinforced, whereas in the presence of DIFFERENT, choosing @ @ @ was reinforced. In this way, training established the functions of TRUE and FALSE for the arbitrary shapes @ @ @ and ***, respectively. Subsequently, these stimuli will be referred to as TRUE and FALSE.

Stage 4(a \& b): Training responding to relations between relations. At the start of each trial, the eight-chambered box appeared $2 \mathrm{~cm}$ above the bottom of the screen (see Figure 2, lower panel). One second later, the contextual cue, either SAME or DIFFERENT, appeared approximately 2 $\mathrm{cm}$ above the center of the first stimulus. One second after that, the sample appeared approximately $3 \mathrm{~cm}$ above the contextual cue. The sample, which may be seen in Figure 2 (lower panel), consisted of a box, approximately $9 \mathrm{~cm}$ by $5 \mathrm{~cm}$, containing two further boxes, both approximately $4 \mathrm{~cm}$ by $4 \mathrm{~cm}$, each of which contained two colored shapes. One second after the appearance of the sample, TRUE and FALSE appeared in synchrony in the top corners of the screen.

On each trial, the sample was a box containing two boxes, each of which contained either two same-colored or two differently colored shapes. If both boxes contained two same-colored shapes, then the relation between the relations in the boxes was one of sameness. If both boxes contained two differently colored shapes, then the relation between the relations in the boxes was also one of sameness. However, if one box contained two samecolored shapes and the other box contained two differently colored shapes, then the relation between the relations in the boxes was one of difference. If the relation between the two relations was the same, then in the presence of SAME, choosing TRUE was reinforced, whereas in the presence of DIFFERENT, choosing FALSE was reinforced. If the relation between the two relations was different, then in the presence of SAME, choosing FALSE was reinforced, whereas in the presence of DIFFERENT, choosing TRUE was reinforced. In this way, subjects were trained to respond to relations between (nonarbitrary color) relations.

Stage 5: Introducing nonsense syllables into the test for responding 
to relations between relations. At the start of each trial, the eightchambered box appeared $2 \mathrm{~cm}$ above the bottom of the screen. During this stage, the box contained nonsense syllables superimposed upon colored shapes (see Figure 3). One second later, the contextual cue, either SAME or DIFFERENT, appeared approximately $2 \mathrm{~cm}$ above the center of the chambered box. One second after that, the sample appeared approximately $3 \mathrm{~cm}$ above the contextual cue. The sample was similar to that which appeared in Stage 4, except that each of the smaller boxes contained two nonsense syllables (rather than colored shapes). One second after the appearance of the sample, TRUE and FALSE appeared in synchrony in the top corners of the screen.

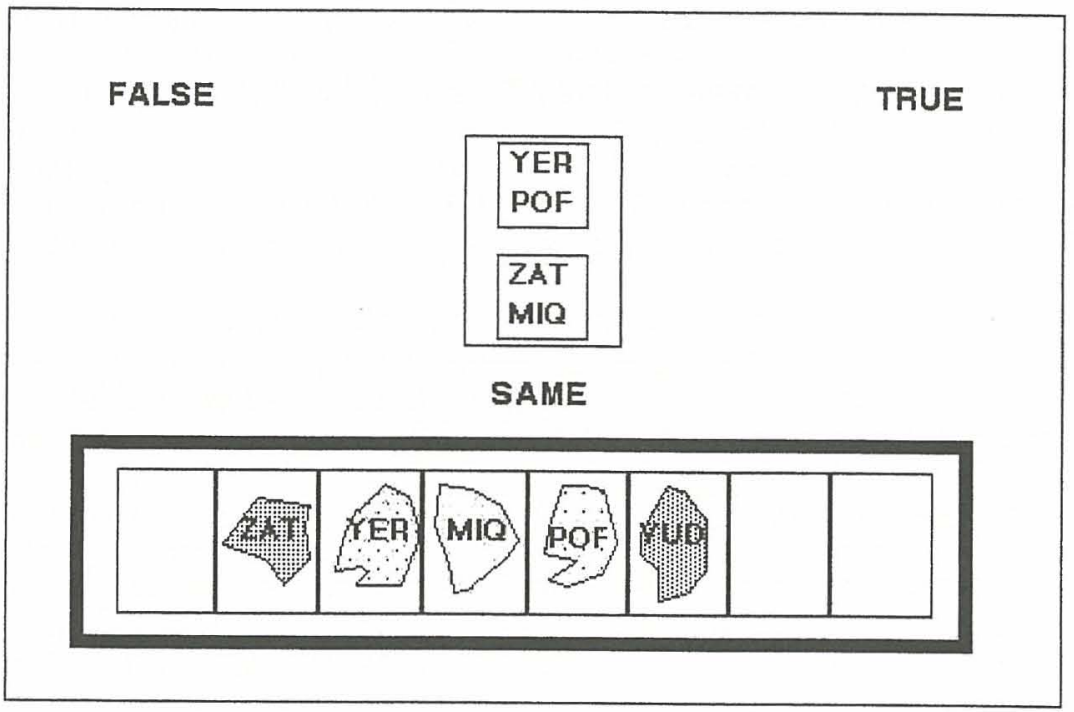

Figure 3. A trial-type representative of Stage 5 testing. The SAME / DIFFERENT, YES, and NO contextual cues were arbitrary shapes, but are shown here as English words for ease of communication.

Based on spatial contiguity, it was predicted that each of the nonsense syllables appearing in the chambers of the bottom box should acquire the color functions of the shapes upon which they were superimposed. In addition, because four of the nonsense syllables appearing in the bottom box appeared also in the box in the center of the screen, it was predicted that subjects would respond to these four nonsense syllables as functionally equivalent to those particular colors. Subsequently, subjects would be expected to respond in accordance with relations between relations in the same manner as in the previous stage.

Stages 6 and 7: Relating nonarbitrary relations based on arbitrary relations of mutual entailment. At the start of each trial, the eightchambered box appeared $2 \mathrm{~cm}$ above the bottom of the screen (see Figure 4). During this stage, a number of the central chambers of the box 
FALSE

TRUE

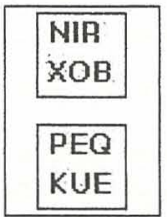

SAME

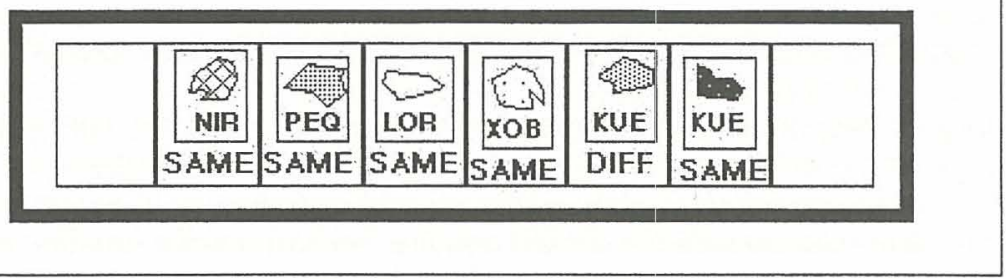

TRUE

FALSE

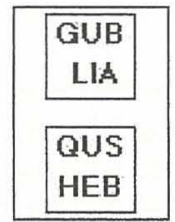

\section{DIFFERENT}

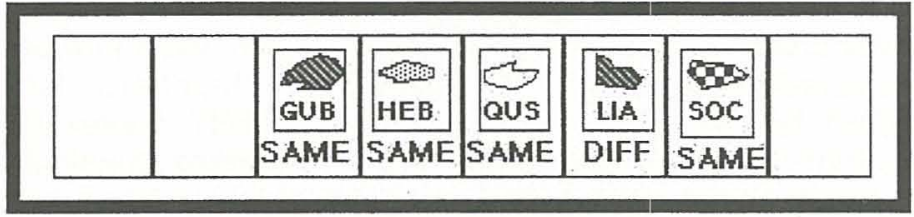

Figure 4. Upper panel: A trial-type representative of Stage 6, which tested for the relating of nonarbitrary relations based on arbitrary relations of mutual entailment. In Stage 6 trialtypes involving difference cues, the nonsense syllable in a relation of different with a colored shape in one chamber always appeared in another chamber in a relation of same with a differently colored shape. This pairing helped to establish responding in accordance with the contextual cue of difference in the chambered box (see text).

Lower panel: A trial-type representative of Stage 7, which tested for the relating of nonarbitrary relations based on arbitrary relations of mutual entailment. This stage differed from Stage 6 only in that there was no pairing of same and different relations in the bottom (chambered) box (see text). 
contained the following elements: a contextual cue (i.e., either SAME or DIFFERENT) at the bottom of the chamber and a box measuring approximately $4 \mathrm{~cm}$ by $5 \mathrm{~cm}$, containing a nonsense syllable and a colored shape, at the top of the chamber. One second later, a contextual cue, either SAME or DIFFERENT, appeared approximately $2 \mathrm{~cm}$ above the center of the chambered box. One second after that, the sample appeared approximately $3 \mathrm{~cm}$ above the contextual cue. The sample was similar to that which appeared in Stage 4. One second after the appearance of the sample, TRUE and FALSE appeared in synchrony in the top corners of the screen.

In this stage, subjects were required to observe the two relata (colored shape and nonsense syllable) and the contextual cue in each of the occupied chambers of the eight-chambered box before looking at the images that appeared above this display. Based on an experimental history of responding in the presence of the SAME and DIFFERENT cues, it was predicted that the functions of the nonsense syllables should transform according to specific patterns. For example, in the presence of SAME, a nonsense syllable should acquire the same color function as the shape over which it appeared. Furthermore, in the presence of DIFFERENT, a nonsense syllable should acquire a function of 'different color from the shape over which it appeared.' After the nonsense syllables acquired these functions, it was predicted that subjects should respond in accordance with relations between relations in the same manner as in previous stages.

The reader should note that there were a number of trials in which one of the chambers contained a DIFFERENT cue. It was predicted that subjects would respond to the nonsense syllable inside the box above the cue as "different from" the colored shape inside the same box. In order to facilitate control by the DIFFERENT cue, during Stage 6 there was another chamber that contained a SAME cue underneath a box that contained a different-colored shape, but the same nonsense syllable as in the former chamber (see Figure 4). Extensive pilot work indicated that the simultaneous presence of these two chambers facilitated discrimination between the SAME and DIFFERENT contextual cues within the eight-chambered box. For illustrative purposes, consider Figure 4 (upper panel); the two filled chambers furthest to the right contain the same nonsense syllable (i.e., KUE) with different colored shapes. One chamber contains a SAME cue and the other contains a DIFFERENT cue. The two respective cues indicate that KUE should be responded to as the same as one color, but different from the other.

Stage 7 (see Figure 4, lower panel) was exactly the same as Stage 6 , except that on trials in which there was a chamber with a DIFFERENT cue, there was no counterpart SAME-cue chamber. In effect, the relational network did not specify what color should transfer to the nonsense syllable, only what color should not transfer.

Stage 8: Relating nonarbitrary relations based on arbitrary relations of combinatorial entailment. This stage was identical to Stage 7, except 
that all chambers in the eight-chambered box were filled on each trial. Furthermore, subjects could only produce experimenter-designated correct responses by combining the contextually controlled relations

NO

YES

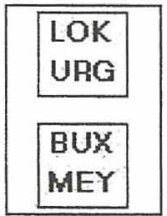

SAME

\begin{tabular}{|c|c|c|c|c|c|c|c|}
\hline GEF & ORD & GEF & LOK & HEY & WAK & BAB & URG \\
\hline BUX & & 6 & $B A B$ & ORD & & & WAK \\
\hline SAME & SAME & SAME & SAME & SAME & SAME & SAME & DIFF \\
\hline
\end{tabular}

No

YES

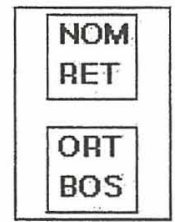

SAME

\begin{tabular}{|c|c|c|c|c|c|c|c|}
\hline $\begin{array}{l}\text { BOS } \\
\text { NEK }\end{array}$ & $\begin{array}{l}\text { ORT } \\
\text { LEL }\end{array}$ & \begin{tabular}{|c|} 
NEK \\
总
\end{tabular} & $\begin{array}{l}\text { RET } \\
X \mathrm{AL}\end{array}$ & \begin{tabular}{|l} 
LEL \\
颌 \\
\end{tabular} & $\begin{array}{l}\mathrm{XAL} \\
6\end{array}$ & $\begin{array}{ll}\mathrm{CHO} \\
\end{array}$ & \begin{tabular}{|l|}
$\mathrm{MOH}$ \\
$\mathrm{CHU}$
\end{tabular} \\
\hline SAME & SAME & SAME & SAME & SAME & SAME: & SAME & DIFF \\
\hline
\end{tabular}

Figure 5. Upper panel: A trial-type representative of Stage 8, which tested for the relating of (nonarbitrary color) relations based on arbitrary relations of combinatorial entailment (analogical responding). This stage employed foils to ensure that subjects were in fact responding in accordance with relations of difference involving nonsense syllables.

Lower panel: A trial-type representative of Stage 9, which tested for the same type of responding (i.e., analogical responding) as in Stage 8, but in which the colored shapes and nonsense syllables were completely novel. 
across two chambers. In Figure 5 (upper panel), for example, the subject had to combine the relations specified in the first and third chambers (reading from left to right) in order to derive the color of BUX (via GEF).

Similar to Stages 6 and 7 there were a number of trials in which one of the chambers contained a DIFFERENT cue. However, it is important to note that there were a number of trials in which this cue, though present, was not a part of the analogical relational network (see Figure 5, upper panel, for example). Such trial types were employed to ensure that subjects were not responding to DIFFERENT simply by doing the opposite of what they would otherwise do, but were actually responding in accordance with the complete relational network.

Stage 9: Test for analogical responding with novel colors and shapes. This stage was identical to the previous stage, except that it involved completely novel colors, shapes, and nonsense syllables. Subjects received 24 trials, which, if completed successfully, would provide a demonstration of 24 completely novel examples of analogical responding as defined by Relational Frame Theory.

\section{Results}

Table 1 presents the number of trials required by each subject to complete training and testing for Stages 1, 2, 3, and 4, and to complete testing for Stages 5, 6, 7, 8, and 9. For illustrative purposes, the data for Subject 1 will be described in detail. This subject required a total of 24 trials to complete Stage 1(a), Same/Different training with pictures, and he then passed the Stage 1 (b) test with 12/12 correct. He needed a total of 24 trials to complete Stage 2(a), Same/Different training with colors, and he then passed the Stage 2(b) test with 12/12 correct. The subject required 24 trials to complete Stage 3(a), TRUE/FALSE training, and then he passed the Stage 3(b) test with 12/12 correct. The subject was then exposed to 120 Stage 4(a) training trials (relating nonarbitrary relations to nonarbitrary relations), before finishing the experimental session for the day. On his return, the subject was reexposed to all previously received stages of training and testing and successfully completed Stage $4(a$ \& $b)$ and then Stage 5 (introduction of nonsense syllables into the test for relating relations) with 12/12 correct, before the end of that day's session. On his return, the subject was once more reexposed to all previous stages of training and testing, which he successfully completed. He failed on his first exposure to Stage 6 (relating relations based on arbitrary relations of mutual entailment) and was thus reexposed to all previous stages, which he successfully completed. He then failed Stage 6 once again, and was thus reexposed to all previous stages, which he again successfully completed. On his third exposure to Stage 6 he passed, and then he immediately passed Stage 7 (removal of redundant SAME cue from Stage 6), Stage 8 (relating relations based on arbitrary relations of combinatorial entailment), and finally Stage 9 (analogical responding with novel colors and shapes). 
As can be seen through careful inspection of Table 1, all 5 subjects successfully completed all nine stages of the experimental protocol. Only 1 other subject (Subject 5) required more than one experimental session before completing it, and Subjects 2 and 3 completed it without failing any of the test stages. These results provide strong support for the REPbased model of analogy and demonstrate the power of this procedure to generate an in-principle infinite number of complex relational responses.

Table 1

Numbers of Training Trials in Stages 1(a), 2(a), 3(a), and 4(a) and Numbers of Correct Responses in Testing Trials Stages 1(b), 2(b), 3(b), 4(b), 5, 6, 7, 8, and 9 for All Subjects

\begin{tabular}{|c|c|c|c|c|c|c|c|c|c|c|c|c|c|}
\hline $\begin{array}{l}\text { Sub } \\
\text { No. }\end{array}$ & $\begin{array}{l}\text { St. } \\
1 \text { (a) }\end{array}$ & $\begin{array}{c}\text { St. } \\
1 \text { (b) }\end{array}$ & $\begin{array}{l}\text { St. } \\
\text { 2(a) }\end{array}$ & $\begin{array}{l}\text { St. } \\
\text { 2(b) }\end{array}$ & $\begin{array}{l}\text { St. } \\
3(a)\end{array}$ & $\begin{array}{l}\text { St. } \\
3(b)\end{array}$ & $\begin{array}{l}\text { St. } \\
\text { 4(a) }\end{array}$ & $\begin{array}{l}\text { St. } \\
4 \text { (b) }\end{array}$ & $\begin{array}{l}\text { St. } \\
5\end{array}$ & $\begin{array}{l}\text { St. } \\
6\end{array}$ & $\begin{array}{l}\text { St. } \\
7\end{array}$ & $\begin{array}{c}\text { St. } \\
8\end{array}$ & $\begin{array}{l}\text { St. } \\
9\end{array}$ \\
\hline \multirow[t]{5}{*}{1} & 24 & $12 \mathrm{P}$ & 24 & $12 P$ & 24 & $12 \mathrm{P}$ & 120 & & & & & & \\
\hline & 12 & $12 P$ & 12 & $12 P$ & 12 & $12 \mathrm{P}$ & 144 & $12 \mathrm{P}$ & $12 P$ & & & & \\
\hline & 12 & $12 P$ & 12 & $12 \mathrm{P}$ & 12 & $12 \mathrm{P}$ & 12 & $12 \mathrm{P}$ & $12 \mathrm{P}$ & $8 F$ & & & \\
\hline & 12 & $12 P$ & 12 & $12 \mathrm{P}$ & 12 & $12 P$ & 12 & $12 \mathrm{P}$ & $12 P$ & $8 F$ & & & \\
\hline & 12 & $12 \mathrm{P}$ & 12 & $12 \mathrm{P}$ & 12 & $12 \mathrm{P}$ & 12 & $12 \mathrm{P}$ & $12 P$ & $12 \mathrm{P}$ & $12 \mathrm{P}$ & $12 \mathrm{P}$ & $24 \mathrm{P}$ \\
\hline 2 & 24 & $12 \mathrm{P}$ & 24 & $12 \mathrm{P}$ & 12 & $12 \mathrm{P}$ & 120 & $12 P$ & $12 \mathrm{P}$ & $12 \mathrm{P}$ & $12 P$ & $12 \mathrm{P}$ & $24 \mathrm{P}$ \\
\hline 3 & 48 & $12 \mathrm{P}$ & 24 & $12 P$ & 24 & $12 \mathrm{P}$ & 144 & $12 P$ & $12 \mathrm{P}$ & $12 \mathrm{P}$ & $12 \mathrm{P}$ & $12 \mathrm{P}$ & $24 \mathrm{P}$ \\
\hline \multirow[t]{2}{*}{4} & 12 & $12 \mathrm{P}$ & 12 & $12 \mathrm{P}$ & 12 & $12 \mathrm{P}$ & 24 & $12 \mathrm{P}$ & $12 \mathrm{P}$ & $12 \mathrm{P}$ & $12 \mathrm{P}$ & $3 F$ & \\
\hline & 12 & $12 \mathrm{P}$ & 12 & $12 \mathrm{P}$ & 12 & $12 \mathrm{P}$ & 12 & $12 \mathrm{P}$ & $12 \mathrm{P}$ & $12 \mathrm{P}$ & $12 \mathrm{P}$ & $12 \mathrm{P}$ & $24 \mathrm{P}$ \\
\hline & 24 & $12 \mathrm{P}$ & 12 & $12 P$ & 24 & $12 \mathrm{P}$ & 60 & $12 \mathrm{P}$ & & & & & \\
\hline & 12 & $12 \mathrm{P}$ & 12 & $12 P$ & 12 & $12 P$ & 12 & $12 \mathrm{P}$ & $12 P$ & $8 \mathrm{~F}$ & & & \\
\hline & 12 & $12 \mathrm{P}$ & 12 & $12 \mathrm{P}$ & 12 & $12 \mathrm{P}$ & 12 & $12 P$ & $12 \mathrm{P}$ & $12 \mathrm{P}$ & $12 \mathrm{P}$ & $12 P$ & $24 \mathrm{P}$ \\
\hline
\end{tabular}

\section{Discussion}

The results of this study are that 5 subjects successfully completed the REP model of analogy. The subjects' behavior was successfully brought under SAME and DIFFERENT, and TRUE and FALSE, contextual control. The subjects were then trained to respond in accordance with relations between relations. Following this training, subjects were then provided with a series of increasingly complex tests involving relating relations, and by Stage 9 of the current protocol they readily demonstrated 24 completely novel instances of responding in accordance with analogical relations. Overall, these results provide strong support for the REP model of analogy.

Subjects 2 and 3 passed through each stage of training and testing with no need for any reexposure to earlier stages. Given the complexity of the final performances involved, these outcomes might seem unusual. However, the protocol reported herein evolved over the course of a long series of pilot studies involving sizeable numbers of subjects. Furthermore, it could be argued that once the subjects' behavior came 
under the control of the relevant contextual cues, the performances simply involved the same overarching functional class of arbitrarily applicable relational responding during each subsequent stage of the protocol. Insofar as this was the case, the current study met its primary objective- to develop a procedure that successfully and readily produces responding in accordance with relations between relations. Having done so, subsequent research may now employ this procedure in order to examine the effects of other variables on this behavioral class. For example, one direction for future study could involve developing programs for establishing the relating of relations when this performance is found to be absent in the behavioral repertoires of particular populations (Alexander, White, Haensly, \& Crimmins-Jeanes, 1987; Alexander, Willson, White, \& Fuqua, 1987; Masterson, Evans, \& Aloia, 1993).

One stage of the protocol that some subjects failed more than once was Stage 6, which involved the introduction of both SAME and DIFFERENT contextual cues into the eight-chambered box for the first time. Initially, Stage 6 was omitted (i.e., after completing Stage 5, subjects were exposed immediately to what is now called Stage 7). However, under these conditions, many pilot subjects did not come under the control of the SAME and DIFFERENT cues. Instead of responding in accordance with the DIFFERENT cue, these earlier subjects tended to respond to stimuli in the same box (i.e., the nonsense syllable and the colored shape) as the same as each other. One likely reason for this was that the subjects' prior history with respect to the eight-chambered box (i.e., Stage 5) involved treating spatially contiguous stimuli as the same as each other. Indeed, a number of recent studies have reported that spatial contiguity readily leads to the formation of equivalence classes in both adults and children (see Leader \& Barnes, 1996; Leader, BarnesHolmes, \& Smeets, 2000). Thus, spatial contiguity likely exerted greater control over responding with respect to the eight-chambered box than did the contextual cue of DIFFERENT (and possibly SAME). In order to gain control of responding by SAME and DIFFERENT in the context of the eight-chambered box, Stage 6 of the current protocol was introduced, in which the chamber containing the DIFFERENT cue had a counterpart chamber containing the same nonsense syllable with a different-colored shape and a SAME cue. As explained previously, the presence of these two chambers "highlighted" that the presence of a DIFFERENT cue in a chamber indicates that the color functions of the shape should not transfer to the nonsense syllable that appears in the same chamber. The responding of 3 of the subjects in this study came under the control of SAME and DIFFERENT immediately upon their exposure to Stage 6, but for 2 subjects, responding came under the control of SAME and DIFFERENT after a number of exposures to this stage. Future research may further explore the relative controlling power of spatial contiguity versus previously trained contextual cues.

As is the case with any model of analogical reasoning, the current REP model likely fails to capture certain properties of this phenomenon. 
However, many if not most of the properties not captured in the current model could be addressed successfully, both conceptually and empirically, in subsequent research. In what follows, we will attempt to show how this might be achieved.

Stewart, Barnes-Holmes, Roche, et al. (2001) reported a model of analogy as relating relations based on nonarbitrary similarity. The current model also demonstrated the relating of relations based on nonarbitrary similarity. However, whereas in the Stewart et al. model the relation between relations was always an equivalence relation, in the current model, many of the trials involved responding in accordance with a relation of difference between relations. For example, on many trials, subjects had to affirm that an arbitrarily applicable relation of difference was applicable between two arbitrary relations, based on the nonarbitrary (color) relations between the shapes on the screen. Because analogy does not typically involve a relation of difference between relational networks, it could be argued that such performances are examples of highly unusual relational networks that arise rarely outside the context of a laboratory-generated artificial language, such as that created in the present experiment. The demonstration of exannples of novel or unusual arbitrarily applicable relational responding such as this, alongside the types of relational responding that might ordinarily characterize language, is a particularly interesting development. It shows the potential of the REP as a means by which we may explore and test the boundaries of language, and by which we may even extend the boundaries of normal linguistic performance within the context of the laboratory (e.g., Ferreira, Christianson, \& Hollingworth, 2001; Gibson \& Thomas, 1999).

The present REP model of analogy was a very basic one in that it involved the discrimination of formal or nonarbitrary similarity along only one physical dimension, namely that of color. Analogies in natural language typically involve formal or nonarbitrary similarities along numerous dimensions. Perhaps a more ecologically valid REP model of analogy, therefore, would involve multidimensional stimuli, from which a range of formal or physical properties might be abstracted (see e.g., Stewart, Barnes-Holmes, Roche, et al., 2001). In the present model, pictures of multidimensional stimuli were used in the first stage, Stage 1(a \& b), in order to establish contextual cues for SAME and DIFFERENT, but color was the only physical dimension that was relevant in later stages. $A$ subsequent model of analogy might involve the use of such multidimensional picture stimuli throughout the experiment.

There are many other conceptual and theoretical issues that have emerged from the current interpretation of analogy in terms of relating relations (see Stewart, Barnes-Holmes, Roche, et al., 2001; Stewart, Barnes-Holmes, Hayes, \& Lipkens, 2001). The success of the current REP model provides an opportunity for the ernpirical analysis of these issues. Such work may well be important in that analogy and other related psychological phenomena, such as metaphor, have received little empirical attention within the behavior-analytic research community. 


\section{References}

ALEXANDER, P. A., WHITE, C. S., HAENSLY, P. A., \& CRIMMINS-JEANES, M. (1987). Training in analogical reasoning. American Educational Research Journal, 24, 387-404.

ALEXANDER, P. A., WILLSON, V. L., WHITE, C. S., \& FUQUA, J. D. (1987). Analogical reasoning in young children. Journal of Educational Psychology, $79,401-408$.

BARNES, D. (1994). Stimulus equivalence and relational frame theory. The Psychological Record, 44, 91-124.

BARNES, D., HEGARTY, N., \& SMEETS, P. M. (1997). Relating equivalence relations to equivalence relations: A relational framing model of complex human functioning. The Analysis of Verbal Behavior, 14, 57-83.

BARNES, D., \& HOLMES, Y. (1991). Radical behaviorism, stimulus equivalence, and human cognition. The Psychological Record, 41, 19-31.

CULLINAN, V. A., BARNES, D., \& SMEETS, P. M. (1998). A precursor to the relational evaluation procedure: Analyzing stimulus equivalence. The Psychological Record, 48, 121-145.

CULLINAN, V. A., BARNES-HOLMES, D., \& SMEETS, P. M. (2000). A precursor to the relational evaluation procedure: Analyzing stimulus equivalence II. The Psychological Record, 50, 467-492.

DYMOND, S., \& BARNES, B. (1994). A transfer of self-discrimination response functions through equivalence relations. Journal of the Experimental Analysis of Behavior, 62, 251-267.

DYMOND, S., \& BARNES, D. (1995). A transformation of self-discrimination response functions in accordance with the arbitrarily applicable relations of sameness, more-than, and less-than. Journal of the Experimental Analysis of Behavior, 64, 163-184.

DYMOND, S., \& BARNES, D. (1996). A transformation of self-discrimination response functions in accordance with the arbitrarily applicable relations of sameness and opposition. The Psychological Record, 46, 271-300.

ELIASMITH, C., \& THAGARD, P. (2001). Integrating structure and meaning: A distributed model of analogical mapping. Cognitive Science, 25(2), 245-286.

EYSENCK, M. W., \& KEANE, M. T. (2000). Cognitive psychology: A student's handbook (4th ed.) New York: Psychology Press.

FERREIRA, F., CHRISTIANSON, K., \& HOLLINGWORTH, A. (2001). Misinterpretations of garden-path sentences: Implications for models of sentence processing and reanalysis. Journal of Psycholinguistic Research, 30(1), 3-20.

FORBUS, K. D., FERGUSON, R. W., \& GENTNER, D. (1994). Incremental structure mapping. In A. Ram \& K. Eiselt (Eds.), Proceedings of the Sixteenth Annual Conference of the Cognitive Science Society (pp. 313318). Atlanta, GA: Lawrence Erlbaum Associates.

GIBSON, E., \& THOMAS, J. (1999). Memory limitations and structural forgetting: The perception of complex ungrammatical sentences as grammatical. Language \& Cognitive Processes, 14(3), 225-248.

HAYES, S. C. (1991). A relational control theory of stimulus equivalence. In L. J. Hayes \& P. N. Chase (Eds.), Dialogues on Verbal Behavior (pp. 19-40). Reno, NV: Context Press.

HAYES, S. C. (1994). Relational frame theory: A functional approach to verbal events. In S. C. Hayes, L. J. Hayes, M. Sato, \& K. Ono (Eds.), Behavior analysis of language and cognition (pp. 9-30). Reno, NV: Context Press. 
HAYES, S. C., \& BARNES, D. (1997). Analyzing derived stimulus relations requires more than the concept of stimulus class. Journal of the Experimental Analysis of Behavior, 68, 235-270.

HAYES, S. C., BARNES-HOLMES, D., \& ROCHE, B. (2001). Relational frame theory: A post Skinnerian approach to language and cognition. New York: Plenum Press.

HOLYOAK, K. J., \& THAGARD, P. (1989). Analogical mapping by constraint satisfaction. Cognitive Science, 13(3), 295-355.

HUMMEL, J. E., \& HOLYOAK, K. J. (1997). Distributed representations of structure: A theory of analogical access and mapping. Psychological Review, 104(3), 427-466.

KEANE, M. T., LEDGEWAY, T., \& DUFF, S. (1994). Constraints on analogical mapping: A comparison of three models. Cognitive Science, 18(3), 387-438.

LEADER, G., \& BARNES, D. (1996). Establishing equivalence relations using a respondent-type training procedure. The Psychological Record, 46, 685-706.

LEADER, G., BARNES-HOLMES, D., \& SMEETS, P. (2000). Establishing equivalence relations using a respondent-type training procedure III. The Psychological Record, 50, 63-79.

MASTERSON, J. J., EVANS, L. H., \& ALOIA, M. (1993). Verbal analogical reasoning in children with language-learning disabilities. Journal of Speech \& Hearing Research, 36(1), 76-82.

ROCHE, B., \& BARNES, D. (1996). Arbitrarily applicable relational responding and sexual categorization: A critical test of the derived difference relation. The Psychological Record, 46, 451-475.

ROCHE, B., STEWART, I., \& BARNES-HOLMES, D. (1999). PsyScope: An easyto use graphical system for designing and controlling equivalence experiments. Experimental Analysis of Human Behavior Bulletin, 17, 5-7.

SKINNER, B. F. (1957). Verbal behavior. New York: Appleton-Century-Crofts.

SKINNER, B. F. (1989). Recent issues in the analysis of behavior. Columbus: Merrill Publishing.

STEELE, D., \& HAYES, S. C. (1991). Stimulus equivalence and arbitrarily applicable relational responding. Journal of the Experimental Analysis of Behavior, 56, 519-555.

STEWART, I., BARNES-HOLMES, D., HAYES, S. C., \& LIPKENS, R. (2001). Relations among relations: Analogies, metaphors and stories. In S. C. Hayes, D. Barnes-Holmes, \& B. Roche (Eds.), Relational frame theory: A post-Skinnerian account of human language and cognition (pp. 73-86). New York: Plenum Press.

STEWART, I., BARNES-HOLMES, D., ROCHE, B., \& SMEETS, P. M. (2001). Generating derived relational networks via the abstraction of common physical properties: A possible model of analogical reasoning. The Psychological Record, 51, 381-408.

STEWART, I., BARNES-HOLMES, D., ROCHE, B., \& SMEETS, P. M. (2002). A functional analytic model of analogy. Journal of the Experimental Analysis of Behavior, 78, 375-396.

VOSNIADOU, S., \& ORTONY, O. (1989). Similarity and analogical reasoning. New York: Cambridge University Press. 


\section{APPENDIX}

Instructions for Stages 1(a \&b) and 2( $a \&$ \& b)

In a moment some images will appear on this screen. First, a box will appear at the bottom of the screen. Next, another image will appear above that. Then, another image will appear above that, and finally, two images will appear in the corners at the top of the screen. You should look first at the image that appears above the box. Next, look at the image that appears above that. Then, having looked at these two images, choose one of the two images in the top corners of the screen. Click the mouse when you are ready to begin.

\section{Instructions for Stages 3(a \& b) and 4(a \& b)}

In a moment some images will appear on this screen. First, a box will appear at the bottom of the screen. Next, another image will appear above that. Then, another image will appear above that, and finally, two images will appear in the corners at the top of the screen. You should look first at the image that appears above the box. Next, look at the image that appears above that. Then, having looked at these two images, choose one of the two images in the top corners of the screen. Click the mouse when you are ready to begin.

Instructions for Stages 5, 6, 7, 8, and 9

In a moment some images will appear on this screen. First, a box will appear at the bottom of the screen. Next, another image will appear above that. Then, another image will appear above that, and finally, two images will appear in the corners at the top of the screen. You should look first at the box. Then look at the image that appears above the box, and then at the image that appears above that, and then, having looked at these images, choose one of the two images in the top corners of the screen. Click the mouse when you are ready to begin. 